\title{
La enseñanza derecho civil a través del elemento audiovisual en titulaciones no jurídicas: una innovación docente en la Universitat Politècnica de València ${ }^{1}$
}

Teaching civil law through the audio-visual element in not juridical degrees: a teaching innovation at the Universitat Politècnica de València.

Francisca Ramón Fernández

Profesora Titular de Universidad (Derecho civil).

Escuela Técnica Superior de Ingeniería del Diseño (ETSID).

Universitat Politècnica de València.

E-mail: frarafer@urb.upv.es

\begin{abstract}
Resumen: El presente trabajo reflexiona sobre la metodología para enseñar derecho en una Universidad técnica, en concreto en la Universitat Politècnica de València (España). Más allá de la clase magistral tradicional, nos adentramos en otras fórmulas introduciendo las nuevas tecnologías de la información y comunicación (TICs) para fomentar el aprendizaje y comprensión del estudiante. Se explica la experiencia realizada con un episodio de una serie de ficción para la resolución de un caso práctico jurídico en la asignatura de legislación patrimonial, que se imparte en el Máster Universitario en Conservación y Restauración de Bienes Culturales, en la Facultad de Bellas Artes, de Valencia. El perfil del alumnado es con escasos o nulos conocimientos jurídicos, y se trata de explicar conceptos jurídicos mínimos que les pueden servir en la práctica profesional como futuro conservador o restaurador de bienes culturales. Se concluye con el análisis de la encuesta realizada por los alumnos, en la que reflejan su percepción sobre la experiencia, y la utilización de diferentes competencias transversales aplicables. Se formulan cinco preguntas siguiendo la escala de Likert; una pregunta para señalar qué competencia se aplica de las 13 indicadas, y una pregunta abierta sobre propuestas de mejora. Los resultados obtenidos confirman la valoración positiva del alumnado en la utilización del elemento audiovisual en el aula, así como su
\end{abstract}

\footnotetext{
${ }^{1}$ Trabajo realizado en el marco del Proyecto I+D+i «Retos investigación» del Programa estatal de I+D+i orientado a los Retos de la Sociedad del Ministerio de Ciencia, Innovación y Universidades: RTI2018097354-B-100 (2019-2022).
} 
consideración como recurso adecuado para la enseñanza de conceptos jurídicos en titulaciones universitarias no jurídicas.

Palabras clave: Docencia, Derecho civil, audiovisual, nuevas tecnologías de la información y comunicación (TICs), experiencia, Universitat Politècnica de València.

\begin{abstract}
The present work thinks about the methodology to teach right in a technical University, in I make concrete in València's Universitat Politècnica (Spain). Beyond the magisterial traditional class, we enter other formulae introducing the new information and communication technologies (ICTs) to promote the learning and comprehension of the student. Is explained the experience realized with an episode of a series of fiction for the resolution of a practical juridical case in the subject of patrimonial legislation, which is given in the Máster Universitario in Conservation and Restoration of Cultural Goods, in the Faculty of Fine arts, of Valencia. The profile of the student is with scanty or void juridical knowledge, and it is a question of explaining to him juridical minimal concepts that can serve them in the professional practice as conservative future or restorer of cultural goods. He concludes with the analysis of the survey realized by the pupils, in which they reflect his perception on the experience, and the utilization of different transverse applicable competitions. Five questions are formulated following Likert's scale; a question to indicate what competences is applied of the 13 indicated ones, and a question opened on offers of improvement. The obtained results confirm the positive valuation of the student in the utilization of the audio-visual element in the classroom, as well as his consideration as resource adapted for the education of juridical concepts in not juridical university degree.
\end{abstract}

Keywords: Teaching, Civil law, audiovisual, new information and communication technologies (ICTs), experience, Universitat Politècnica de València. 


\section{La enseñanza derecho civil a través del elemento audiovisual en}

\section{titulaciones no jurídicas: una innovación docente en la Universitat}

\section{Politècnica de València}

\section{Teaching civil law through the audio-visual element in not juridical degrees: a teaching innovation at the Universitat Politècnica de València.}

\section{Introducción}

La enseñanza del Derecho no está exenta de dificultades, en cualquiera de sus ramas, y cómo no también en el caso del civil. Este camino de aprendizaje se incrementa considerablemente si lo contextualizamos en una Universidad técnica, destinada a formar al alumnado en titulaciones no jurídicas (Cfr. Navarro y Melero, 2018).

La docencia del Derecho civil en cualquiera de las ingenierías que se imparten en la Universitat Politècnica de València, o en titulaciones de máster como es el caso del que se imparte en la Facultad de Bellas Artes referente a la conservación y restauración de bienes culturales o en la Escuela Técnica Superior de Ingeniería del Diseño (ETSID) relativo al Máster en Ingeniería Aeronáutica, pero, incluso en el Máster en Gestión Administrativa que se implantó recientemente en la Facultad de Administración y Dirección de Empresa (FADE), en la que se imparte una asignatura denominada Derecho civil II, supone un cambio de perspectiva para cualquier jurista que se adentra en la disciplina. Y ello sucede por varias razones que vamos a exponer de forma breve.

El perfil del alumnado con que nos encontramos difiere muchísimo del que se encuentra en una Facultad de Derecho, y es por ello que la docencia tiene que ser abordada y transmitida de forma distinta, sin significar que se merme la calidad en la misma (Canós y Ramón, 2009).

Podemos observar que las asignaturas que se imparten, por ejemplo, en el área de Derecho civil difieren de las que se pueden impartir en una Facultad de Derecho. En una Universidad técnica es el derecho aplicado a la práctica, y ello supone un hándicap para el docente que se enfrenta a un "público" muy distinto del que se podía encontrar en un aula que enseñe un Grado de Derecho, por ejemplo. Además, de la peculiaridad de las asignaturas, en el caso de que nos encontremos con alguna que sí que tenga una denominación idéntica, por ejemplo, hemos indicado la referencia a Derecho civil II, el contenido hay que adaptarlo al perfil de ese alumno que nunca se va a enfrentar a un 
Tribunal, o realizar una defensa de un cliente, porque no cursa los estudios conducentes al grado de Derecho, sino un Máster especializado en gestión administrativa.

Otro de los problemas que nos encontramos es la utilización de la metodología del alumno para la realización de los trabajos, incluso en la valoración de los trabajos final de grado que pueden realizar sobre tema jurídico en una titulación no jurídica. Es habitual encontrarse con la opinión del tribunal de que el alumno ha hecho "un trabajo bibliográfico". Desde luego, que eso no es así, el alumno no hace un trabajo bibliográfico, sino que el alumno utiliza la bibliografía para la argumentación de la postura que defiende en el trabajo y para conocer la posición de la doctrina sobre el conflicto jurídico objeto de estudio. Ello se debe a que cuando tiene que definir su metodología indica que es el método deductivo, que participamos en parte de esta afirmación, como también se ocupa de señalar la doctrina (Marín, 2017:90):

"En efecto, el método deductivo, utilizado por el sistema judicial en la resolución de las sentencias y quienes participan en él, considera como suficiente el hecho de realizar una exposición argumentativa lógica mediante las principales estructuras del silogismo para llegar a conclusiones inapelables. Sin embargo, este error limita a los estudiantes de Derecho y también a muchos juzgadores a considerar como suficiente la interpretación del conocimiento en base a la simple argumentación legal soportada en los diversos reglamentos y leyes, sin profundizar en las causas sociales, históricas, circunstancias psicológicas o carencias culturales que pudieran influir en el peso de los hechos o los motivos de las conductas, lo que sesga la aplicación normativa a un solo ángulo en el litigio".

No hay que olvidar que una asignatura jurídica en una titulación técnica no goza precisamente de buena prensa. La idea del alumnado que se enfrenta con una asignatura de estas características es que tiene un gran grado de dificultad, que hay que aprenderse todos los conceptos de memoria, y que cambian las leyes de forma constante (Colao, 2016:8). Se impone establecer la necesidad de la creatividad en la docencia, entendida como realiza Camilloni (2019:11): "el pensamiento es efectivamente creativo cuando está enfocado hacia la resolución flexible e imaginativa de problemas".

En el presente trabajo se pretende mostrar una reflexión y una experiencia de lo que supone la docencia del Derecho civil en titulaciones técnicas, y en cómo se ha encaminado a través de los recursos digitales, las nuevas tecnologías de la información 
y la comunicación (TICs) una configuración de la enseñanza del derecho, lejos de las pautas clásicas. Al mismo tiempo, se muestra la experiencia desarrollada con el uso de las TICs en una asignatura no jurídica, mediante el diseño de un caso práctico jurídico con el soporte de un episodio de una serie audiovisual.

\section{Enseñar el Derecho civil con soporte audiovisual: una innovación pionera}

Las formas de enseñanza del Derecho han ido evolucionando. Se ha pasado de la impartición de la tradicional clase magistral a la utilización de elementos audiovisuales para favorecer la comprensión, y conseguir una mayor involucración del alumnado en la experiencia docente (Canós y Ramón, 2007).

tortuga

En el marco de la Convergencia Europea las nuevas tecnologías ocupan un lugar primordial en la docencia que se imparte en las Universidades y desde luego en el ámbito jurídico también se está aplicando. El trabajo autónomo del alumno se ha incrementado considerablemente (Canós y Ramón, 2005) tras la implantación de los nuevos grados y másteres, y la docencia ha experimentado un cambio notable, con el consiguiente cambio de roles docente y discente (Canós et al., 2008) que se observa de forma más palpable en el caso de la docencia inversa.

Como afirman Mercader y Gairín (2017:260):

“[...] el nuevo rol del docente respecto a la sociedad digital aún no está totalmente desarrollado, siendo necesario desarrollar competencias para atender las nuevas demandas de los estudiantes, derivadas de las necesidades actuales".

Al hilo de lo anterior, si bien es cierto que el método del caso ha sido tradicionalmente utilizado en las Facultades de Derecho para que el alumnado aplicara una visión práctica de la disciplina. Ello no ha estado exento de opiniones sobre dicha metodología docente. Así, podemos señalar como precisa Pérez (2013:5):

"Hasta ahora y en la mayoría de las ocasiones, el alumno se limita a la resolución del tradicional «caso práctico» que se le presenta, aplicando con mayor o menor acierto los textos legales, la más reciente línea jurisprudencial y en su caso, acompañando el comentario de alguna lectura doctrinal recomendada sobre la cuestión compleja a tratar. Sin embargo, el estudiante difícilmente se implica e involucra de lleno en el fondo del problema o asunto planteado, 
mermando considerablemente su proceso formativo y la adquisición de ciertas habilidades necesarias para lograr una formación integral”.

La doctrina se ha ocupado someramente sobre la enseñanza del Derecho a través de medios audiovisuales, e incluso siguiendo la máxima de que "El derecho está en todas partes", la particular visión de figuras jurídicas en cuentos infantiles (principalmente en Andersen) (Álvarez, 2006a y b).

El aprendizaje, por ejemplo, a través del cine, la relación cine y derecho (Cordero, Escribano y Martínez, 2014; Ruiz, 2014) ha estado presente en muchas de las innovaciones docentes que se han implementado en las Universidades. Como indica Ruiz (2010: 5) incidiendo en :

"No obstante, el cine sobrepasa esa función de mero divertimento en cuanto se convierte en medio efectivo de comunicación de masas. Su capacidad de sensibilización e inspiración de modelos de comportamiento hace que se transforme en testigo de las historias o relatos que se reproducen en cada momento a través de la pantalla, poniendo de manifiesto pautas de actuación y puntos de vista sobre hechos y actos humanos. La identificación escénica e interiorización o subjetivización de personajes y de contextos sociales, culturales o políticos son un claro indicativo de la dimensión ideológica presente en las narraciones fílmicas".

Si bien es cierto el recurso audiovisual ha sido utilizado, como indica la doctrina, en el ámbito de la docencia semipresencial, más que en la presencial, sobre todo en el ámbito jurídico (Pérez, 2014). No obstante, como hemos indicado anteriormente, con el Espacio Europeo de Educación Superior (EEES) cada vez son más los profesores que incluyen dentro de su metodología docente, en el ámbito jurídico, las nuevas tecnologías (Ramón y Saz, 2004a y b). Se trata, en definitiva, de aplicar innovación docente para centrarse básicamente en el aprendizaje del estudiante, ajustar los contenidos de las materias, diversas formas y técnicas de evaluación (Ramos, Chiva y Gómez, 2017: 39).

Podemos destacar la experiencia positiva reflejada por la Red de investigación sobre docencia en Derecho penal, Derecho penitenciario y Política criminal (Universidades de Alicante, Huelva y Valencia), en la que contribuyen al uso de distintos materiales audiovisuales, a través del sistema de fichas, considerando que el empleo, en este caso 
de películas cinematográficas contribuye a la adquisición de las competencias (Aa.Vv., 2013: 1078). También se ha utilizado por la doctrina a través de lo denominado “píldoras audiovisuales" (Capote, 2015: 71). De igual modo, Cicero (2018: 100) siguiendo a López Catalán (2002:44) consideró que:

"Los materiales multimedia que combinan texto, imágenes y sonidos (gráficos, fotos, voces, vídeos, secuencias de películas) tienen la fuerza de facilitar la «transmisión de ideas y conceptos, incrementa la retención reforzando la idea, facilita el interés y la motivación, hace posible la observación de procesos a escala normal con economía de tiempo y esfuerzo».

Nos proponemos en este apartado reflexionar sobre ese cambio docente que hemos experimentado en la enseñanza del Derecho civil, con la inclusión de elementos audiovisuales para facilitar el autoaprendizaje.

1. Los objetos y módulos de aprendizaje. La utilización de los denominados objetos de aprendizaje, también conocidos como OAs, en el ámbito del derecho civil se ha realizado con éxito en la docencia dentro de la Universitat Politècnica de València.

Se trata de grabaciones de corta duración sobre conceptos relacionados con el derecho civil, que están descontextualizados, es decir, no son ningún tema de ninguna asignatura, y los puede reutilizar cualquier docente interesado. Son en la mayoría de libre acceso y se encuentran en el repositorio institucional de la Universitat Politècnica de València, Riunet: www.riunet.upv.es y en www.media.upv.es

Los módulos de aprendizaje son secuenciaciones de actividades que elabora el profesorado dentro del contexto de una asignatura, y en el que se incluye uno o más objetos de aprendizaje grabados por el mismo profesor o por cualquier otro, y que sirven para realizar una actividad de una asignatura, en un contexto temporal (Ramón, 2014).

2. La utilización de series de animación. A través del desarrollo del PIME se obtuvo como resultado un repositorio de casos prácticos con el soporte de distintas series de animación (Cabedo et al., 2016). Se trata que el alumnado vea un episodio de una serie conocida, y luego resuelva un caso práctico en el que se le plantean una serie de cuestiones relacionadas con el episodio que ha visto previamente, y también sobre la legislación que resulta aplicable. Con ello se consigue un mejor aprendizaje de los 
conceptos jurídicos, ya que el alumnado observa en el episodio situaciones reales o ficticias que están íntimamente relacionadas con la legislación (Aa.Vv., 2016 b y c).

3. La utilización de series de ficción. Al igual que en las series de animación, se elaboró un repositorio de casos prácticos basados en distintas series de ficción (Cabedo et al., 2017). Se trata que el alumnado visione una serie de ficción y después resuelva un caso práctico que está redactado basándose en el indicado episodio. Es la misma idea que se ha expuesto en la serie de animación, pero utilizando series de ficción (Cfr. Aa.Vv, 2016).

4. Los documentales. Del mismo modo se ha elaborado un repositorio de casos prácticos reutilizables basados en documentales para que el alumno resuelva el supuesto, con el visionado previo del documental en cuestión. Además, se fomenta también el aprendizaje del alumnado a través de su elección del caso, de entre los distintos que aparecen en el manual, valorándose si el elegido se ajusta a los contenidos de la asignatura o no (Cfr. Cabedo et al., 2018).

Los recursos indicados, principalmente el repositorio de casos basados en series de animación y ficción han sido también utilizados en la docencia jurídica para poder evaluar las competencias transversales, como es el caso de la CT09. Pensamiento crítico (Aa.Vv., 2016a y 2017a, b y c), con lo que no sólo han servido para la explicación y mejor comprensión de conceptos jurídicos, sino también como elemento evaluatorio utilizado por el docente.

No podemos concluir este apartado del trabajo sin señalar algunos de los inconvenientes que supone la utilización de determinados recursos audiovisuales. Si bien, en el caso de los objetos y módulos de aprendizajes (que ya hemos indicado incorporan varios objetos a su vez) están disponibles de libre acceso en el repositorio institucional de la Universitat Politècnica de València: media.upv.es y cada docente firma un acuerdo de cesión de derechos de autor de obras digitales.

En otros casos, el problema que nos encontramos está relacionado con la propiedad intelectual de las obras. Ya lo señalaron los profesores de la Red mencionada sobre docencia en derecho penal (Aa.Vv., 2013: 1079), siendo una de las soluciones acudir a vídeos o documentales que se encuentran disponibles en Youtube. 


\section{La experiencia aplicada al alumnado de la asignatura Legislación del} patrimonio cultural, en el Máster Universitario de Conservación y Restauración de

\section{Bienes Culturales, en la Facultad de Bellas Artes de Valencia}

Vamos a referenciar la experiencia que se ha realizado en el Máster Universitario en Creación y Restauración de Bienes Culturales de la Universitat Politècnica de Valencia, en la asignatura Legislación del patrimonio cultural y creación de pequeñas empresas, que tiene una carga docente total de $4.5 \mathrm{cr}$, en la parte impartida de legislación, que tiene una carga de 2.5 cr., durante el curso académico 2017.-2018.

Dicha asignatura tiene un perfil de alumnado graduado en Bellas Artes, sin ningún tipo de conocimiento jurídico referente a la materia de patrimonio cultural. Es presencial, se imparte en el primer semestre, y el número de alumnos asciende a 30.

Nos enfrentamos, pues, a distintos problemas en el aula:

-Desmotivación del alumnado, ya que considera que el Derecho es algo farragoso, estudiar artículos de memoria, y que no lo van a aplicar en la práctica.

-Desconocimiento de los principales conceptos jurídicos mínimos, ya que no tienen ninguna formación, no conocen el sistema jurídico español, y no distinguen entre ley orgánica, ni ley ordinaria, por ejemplo.

-Obligatoriedad en cursar la asignatura, de tal forma que no es de elección voluntaria, y deben aprobarla para obtener la titulación de Máster.

Teniendo como escenario un "público poco entregado a priori" se trataba de conseguir motivar y proporcionar al alumnado los conocimientos jurídicos suficientes que pudiera necesitar en el futuro en su actividad profesional. Si bien es cierto que el alumnado nunca va a "ejercer como abogado/a", sí que se va a enfrentar en su actividad como conservador y/o restaurador de bienes a distintos problemas, entre otros:

-Conocer cuál es la legislación que se puede aplicar, teniendo en cuenta las distintas competencias autonómicas, y la pluralidad de legislación aplicables. Para ello debe saber la prelación de fuentes del ordenamiento jurídico español.

-Distinguir los delitos que se pueden cometer en relación con el patrimonio cultural.

-Delimitar el ámbito de responsabilidad civil que puede tener un conservador y/o restaurador en el desarrollo de su actividad. 
Se perseguía lograr que el alumnado:

a) Detectara problemas jurídicos a través del estudio de distintos casos reales que habían despertado atención en los medios de comunicación. Por ejemplo, en años anteriores se había analizado el caso del Cristo de Borja, con la restauración de una persona no profesional y que llegó a ser noticia a nivel mundial.

b) Fuera capaz de aplicar la legislación que resultaba correcta, sin tener que memorizar ninguna norma, pero sí poder localizar los preceptos y saber buscar en los repositorios adecuados las leyes que se tenían que aplicar. Como señala De Lucas-Santos (2017: 248), en su experiencia de innovación, "potenciar el auto-aprendizaje ya que los alumnos deben realizar un trabajo intelectual individual y aprender a buscar fuentes y recursos de información sobre los que fundamentar sus aportaciones”.

c) Conseguir que los alumnos lleguen a un consenso sobre las opciones jurídicas de solución de los distintos problemas jurídicos que se plantean en el caso, en relación al patrimonio cultural: por ejemplo, si se ha infringido alguna norma; si no se han adoptado las medidas oportunas para la conservación del bien...

d) Lograr que trabajen en equipo, además en el aula, evitando que los trabajos los repartan entre ellos y no sea realmente un trabajo en grupo, sino por partes.

Para lograr una participación mayor en el aula, dinamizar la asignatura, evitar que el alumnado cayera en el desánimo y apatía, se elaboró un diseño de actividades que pasamos a indicar:

-La profesora consideró evitar impartir la docencia siguiendo el sistema tradicional de clase magistral, y optó por elaborar mapas conceptuales en la pizarra al principio de las clases. El alumnado podía seguir las unidades didácticas a través del manual elaborado por la profesora: El patrimonio cultural. Régimen legislativo y su protección (Ramón, 2012).

Esta actividad consiguió que el alumnado pudiera seguir un esquema de conceptos de forma fácil, clara y evitando la monotonía del discurso unilateral (Ramón, 2014).

-El visionado de distintos casos prácticos relacionados con el patrimonio que tenían una repercusión en la prensa, que habían sido tratados anteriormente por el alumnado, y que resultaban sumamente didácticos y además ayudaban para el futuro caso que debían elaborar y presentar oralmente en el aula ( 
-La resolución de un caso práctico que debían resolver y que había sido redactado por los miembros del grupo de innovación jurídica RETAJUDOCA (Recursos tecnológicos para el aprendizaje jurídico, la documentación y la comunicación audiovisual), de la Universitat Politècnica de València. En esta asignatura se eligió el caso publicado en el Manual Casos prácticos jurídicos basados en series de ficción (Cabedo et al., 2017), titulado El descubrimiento de un tesoro, basado en la serie Tutankamon.

Para la realización de la actividad podían visionar el episodio a través de youtube, que estaba disponible, con libre acceso.

Para determinar la percepción del alumnado acerca de la utilización de una serie como elemento audiovisual previo para resolver un caso práctico relacionado con la asignatura, se diseñó una encuesta, siguiendo la escala de Likert, en el que debían mostrar su conformidad o disconformidad (5-1) el alumnado, en la que se formulaban las siguientes preguntas y que fue elaborada por el profesor Oltra Gutiérrez, miembro del grupo RETAJUDOCA, y participante en el PIME de la UPV 2017-2018 sobre La utilización de los documentales como herramienta para la evaluación de la competencia transversal CT07. Responsabilidad ética, medioambiental y profesional:

-Me gusta el empleo en aula de elementos audiovisuales de forma previa a la resolución de un caso práctico.

-El vídeo empleado tiene relación con el caso práctico que se ha trabajado.

-He detectado elementos relacionados con la teoría vista en clase en el vídeo empleado.

-El vídeo empleado tiene relación con la competencia transversal de responsabilidad ética, medioambiental y profesional.

-La actividad realizada me facilita comprender algunos contenidos de la asignatura.

A continuación se la consultaba al alumnado qué competencia transversal podría aplicar en la actividad que ha realizado, pudiendo subrayar las que considerase oportuno. Las competencias transversales que se indicaban eran las siguientes:

1. Comprensión e integración.

2. Aplicación y pensamiento práctico.

3. Análisis y resolución de problemas.

4. Innovación, creatividad y emprendimiento. 


\section{Diseño y proyecto.}

6. Trabajo en equipo y liderazgo.

7. Responsabilidad ética, medioambiental y profesional.

8. Comunicación efectiva.

9. Pensamiento crítico.

10. Conocimiento de problemas contemporáneos.

11. Aprendizaje permanente.

12. Planificación y gestión del tiempo.

13. Instrumental específica.

Por último, se formulaba una pregunta abierta referente a cuáles serían las propuestas del alumnado para mejorar la actividad planteada.

La encuesta se pasó a los 30 alumnos de la asignatura, de los que contestaron 23.

\section{Resultados obtenidos en la experiencia}

La percepción del alumnado sobre las preguntas referentes al visionado de la serie de ficción elegida como soporte al caso práctico arrojan unos resultados muy interesantes, como apreciamos en el gráfico 1, y que procederemos a comentar: 


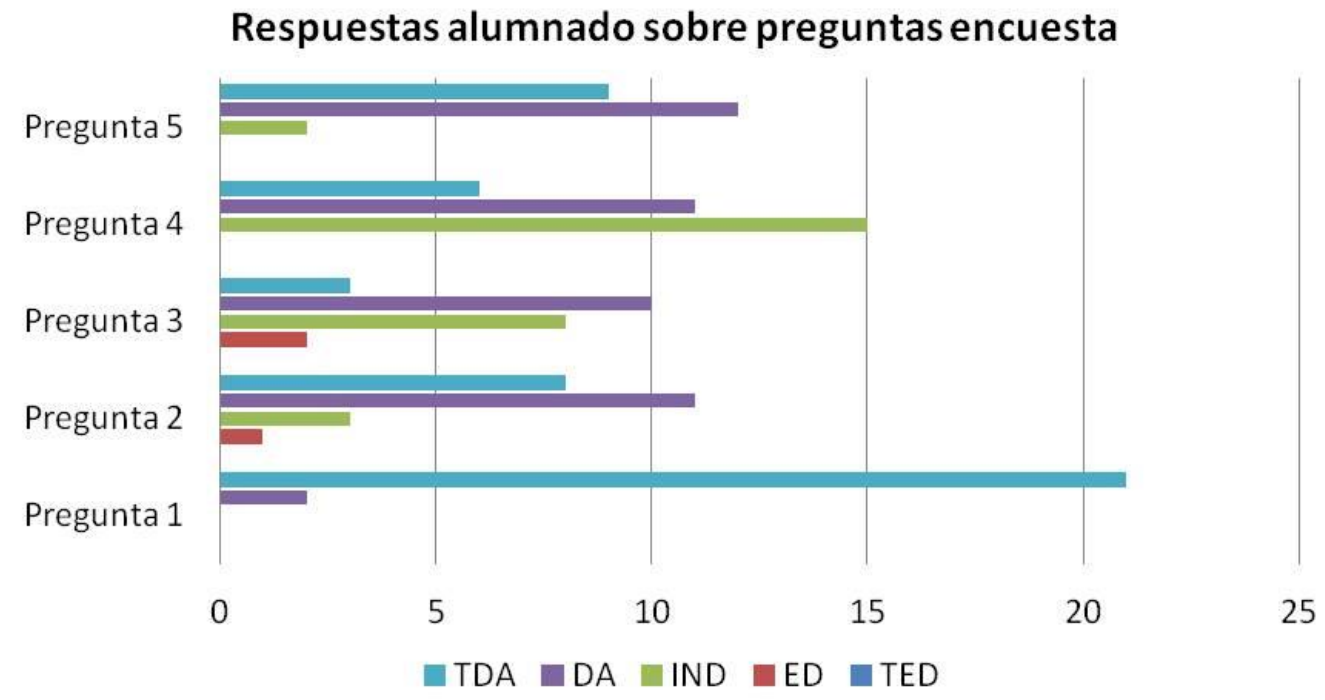

Gráfico 1. Respuestas según escala de Likert. TDA: Totalmente de acuerdo; DA: De acuerdo; IND: Indiferente; ED: En desacuerdo; TED: Totalmente en desacuerdo. Fuente: Elaboración propia

La mayoría de alumnos, un total de 21 respuestas de 23 , se inclinaron por estar totalmente de acuerdo en considerar que les gustaba el empleo en aula de elementos audiovisuales de forma previa a la resolución de un caso práctico. Sólo 2 respondieron que estaban de acuerdo, con la valoración.

Sobre la cuestión relativa a si el vídeo que se empleaba tenía relación con el caso práctico trabajado, la mayoría (11 alumnos) contestaron que estaban de acuerdo; 8 que estaban totalmente de acuerdo, y 3 que les era indiferente.

Acerca de la consulta sobre si se había detectado elementos relacionados con la teoría vista en clase en el vídeo que se ha empleado, sólo dos personas no detectaron elementos; pero la mayoría (10) contestaron que estaban de acuerdo; 3 totalmente de acuerdo, y a 8 les resultó indiferente.

La cuarta pregunta consistía en observar si el alumnado podía relacionar el vídeo con una competencia transversal, en concreto la que se trabaja en relación con la responsabilidad ética, medioambiental y profesional. La contestación a esta pregunta 
coincide con el siguiente apartado de consulta respecto de las competencias transversales que el alumnado consideraba que se reflejaban en el episodio de la serie de ficción utilizada. De tal forma que 11 alumnos estaban totalmente de acuerdo en que se aplicaba la competencia de responsabilidad ética, medioambiental y profesionales, 6 estaban totalmente de acuerdo, y a 15 consideraban que era indiferente.

La última pregunta que se refería a si la actividad realizada de ver el episodio previo de serie de ficción y luego resolver el caso práctico relacionado, les facilitaba la comprensión de algunos contenidos de la asignatura, consideraron la mayoría que estaban de acuerdo (12), totalmente de acuerdo lo consideraron 9, e indiferente, un total de 2.

Por todo ello, extraemos las siguientes conclusiones:

-La mayoría del alumnado considera positivo la utilización de series de ficción como soporte para resolver un caso práctico.

-La mayoría ha sido capaz de detectar elementos jurídicos explicados en el aula con el caso planteado.

-La mayor variación la observamos en la relación con la competencia transversal que nos interesaba conocer si era detectada por el alumnado, ya que la mayoría consideró que era indiferente, pero sí que fueron capaces de considerar que se aplicaban otras competencias transversales, lo cual consideramos positivo, porque la pretensión era no sólo la competencia que se indicaba (responsabilidad ética, medioambiental y profesional), sino que el alumnado se manejara en distintas competencias transversales y ver su aplicación.

Por lo que se refiere a las competencias, el alumnado respondió de diversa forma respecto a qué competencia trabajaba:

En el gráfico 2 podemos ver el número de respuestas, siendo la mayoría la competencia de pensamiento crítico y la menos señalada, por igual, la de innovación, creatividad y emprendimiento y la de diseño y proyecto. 


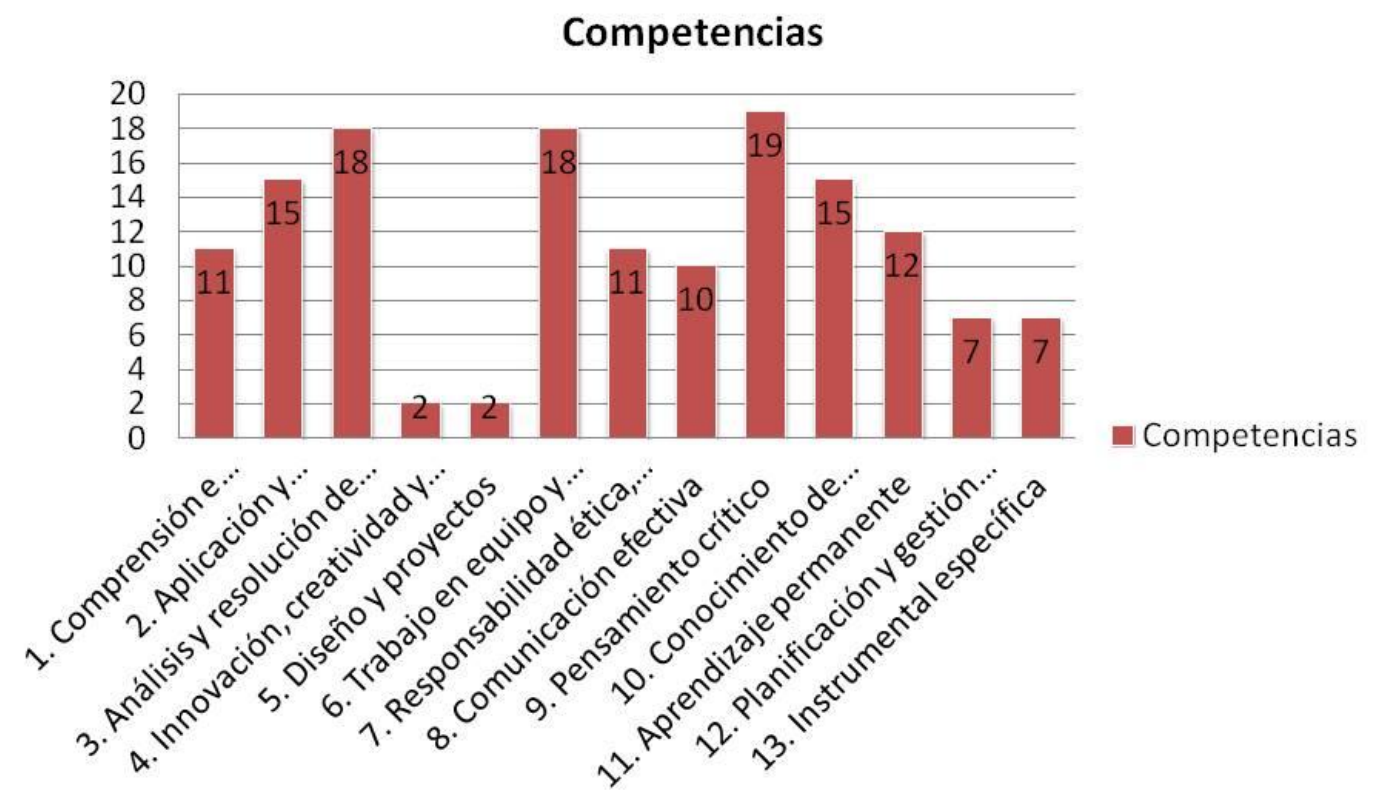

Gráfico 1. Competencias consideradas por los alumnos trabajadas en el caso de la serie Tutankamon. Fuente: elaboración propia

Del análisis consideramos que fueron todas las competencias transversales aplicadas, en mayor o menor medida, y que ninguna competencia fue excluida de ser considerada aplicable.

Respecto a la pregunta abierta, contestaron sólo 7 alumnos de los 23, ya que la mayoría dejaron la respuesta en blanco. Las contestaciones del alumnado fueron las siguientes:

- "Pondría un vídeo que fuera en España, que aplicara la ley estatal".

- “El vídeo podría tener más relación con las preguntas en el sentido que muestra un ejemplo, pero que no tienen el mismo contexto. Por ejemplo, el vídeo tiene como contexto Egipto, mientras que las preguntas son en el caso de España”.

- "Que el vídeo resolviese las dudas planteadas, ya que en este caso el vídeo muestra el problema, pero no la solución y la pregunta se refiere a otro país (España) que en el que ocurre en el vídeo (Egipto).

De todos modos veo bien el vídeo para la comprensión del caso práctico, me parece una buena herramienta didáctica". 
- "Creo que se podrían ofrecer varios casos (relacionados con patrimonio) para que cada alumno pueda escoger el que sea de su interés y/o especialidad y así trabajarlo con más motivación”.

- "Como propuesta de mejora planteo desarrollar preguntas para la actividad que se relacionaran más con el vídeo. Es decir, si leemos lo que se introduce en el manual, no se hace exclusivamente necesario ver el capítulo, si bien contextualiza desde una manera más práctica, no es imprescindible”.

- "Añadir más libros a la biblioteca".

- “Quizás me habría gustado poder tener más tiempo en clase para poder consultar sobre la legislación a la profesora, ya que era un caso difícil de localizar".

Las respuestas del alumnado sobre propuestas de mejora de la actividad, no responden a una percepción objetiva, ya que se centran en impresiones subjetivas sobre el episodio, cuando la pretensión era propuestas objetivas sobre la actividad. Sin embargo, consideramos que nos aportan datos interesantes para mejora de la actividad, en el sentido de diseñar en el futuro otros casos prácticos jurídicos que se puedan contextualizar en series españolas, y que puedan detectar figuras del ordenamiento jurídico español de forma más fácil para el alumnado.

A pesar de que el visionado del documental trataba de un problema que se desarrollaba en Egipto, se le indicó de forma expresa al alumnado que lo que se trataba era que fuera capaz de aplicar la legislación que regulaba el tesoro en el Derecho Español, y en la solución del caso todos los alumnos fueron capaces de indicar que la regulación se contenía en el Código Civil y en la Ley de patrimonio histórico español, con indicación expresa de los artículos que regulaban dicha figura.

Consideramos, pues, que el alumnado hizo el esfuerzo más que loable de poder aplicar a un supuesto que no se desarrollaba en la ficción en nuestro país de aplicar la legislación española, ya que observó de forma clara que se trataba de estudiar el tesoro en una excavación y cuál era el régimen jurídico que se aplicaba al descubridor.

\section{Conclusiones}

La introducción del elemento audiovisual en la enseñanza del derecho civil resulta una experiencia que incrementa la interactividad entre el alumnado (Aa.Vv., 2017).

La innovación docente desarrollada resulta positiva para incrementar la comprensión del alumnado y poder dinamizar el aula (Aa.Vv., 2016d). Se potencia el aprendizaje 
práctico del derecho, superando la percepción de que las asignaturas jurídicas son de carácter áspero y difícil (Urías, 1999).

En el caso de la experiencia mostrada en los resultados del presente trabajo hemos podido observar que:

-El alumnado comprende de forma más amplia los conceptos jurídicos que se explican en el aula contextualizándolo en situaciones reflejadas en series audiovisuales.

-La resolución de un caso práctico resulta más atractiva para el alumnado con el soporte de un audiovisual, más que con el diseño de un tradicional caso práctico en el que el alumno no puede observar visualmente ninguna situación.

-El diseño de casos prácticos jurídicos basándose en series audiovisuales nos permite también al profesorado evaluar las competencias transversales, ya que supone una herramienta idónea para conocer si el alumnado ha sido capaz de observar determinadas situaciones, y aplicar la normativa correspondiente, dependiendo de la competencia que tengamos intención de evaluar.

Como carencia podemos destacar que en el caso de las asignaturas jurídicas en titulaciones técnicas el alumnado no logra adquirir una visión totalmente completa de la disciplina, ya que uno de los recursos que le supone más esfuerzo es la utilización de la jurisprudencia (Verdera, 2014: 20).

\section{Referencias bibliográficas}

Aa. Vv. (2013), “La enseñanza del Derecho penal a través de medios audiovisuales”, en La producción científica y la actividad de innovación docente en proyectos de redes, Universidad de Alicante, Alicante pp. 1068-1082. Disponible en: http://rua.ua.es/dspace/handle/10045/36042 (Consultado el 01 de junio de 2019).

Aa. Vv. (2016), "Uso de fragmentos de películas y series como herramientas de innovación docente. Una experiencia con alumnos de informática", en INRED. II Congreso Nacional de Innovación Educativa y Docencia en Red, Universitat Politècnica de Valencia, València, pp. 592-602. Disponible en: http://ocs.editorial.upv.es/index.php/INRED/INRED2016/paper/viewFile/4430/2000

(Consultado el 25 de mayo de 2019).

Aa.Vv. (2016a), "La evaluación de la competencia transversal trabajo en equipo y liderazgo utilizando las nuevas tecnologías de la información y comunicación (TICs) en 
la Universitat Politècnica de València: experiencia en el caso de series de animación" en Actas del Congreso sobre la educación del siglo XXI, Servicios Académicos Intercontinentales. Universidad de Málaga, Málaga, pp. 259-264. Disponible en: http://www.eumed.net/libros-gratis/actas/2016/educacion/index.htm (Consultado el 07 de enero de 2018).

Aa.Vv. (2016b), "La interactividad y dinamización en el aula mediante la implantación del diseño de casos prácticos jurídicos basados en series de animación”, en I Congreso Internacional online del Uso de las Tic en la sociedad, la educación y la empresa, Libro de resúmenes de $1^{\circ}$ Congreso Internacional online. El uso de las TIC en la sociedad, la educación y la empresa, 3 Ciencias, Área de Innovación y desarrollo, S.L., Alcoy, pp. 103-107.

Aa.Vv. (2016c), "Diseño de casos prácticos jurídicos basados en series de animación. Una innovación docente con soporte audiovisual", en Docencia del Derecho y TIC: innovación y experiencias metodológicas, Huygens Editorial, Barcelona, pp. 123-132.

Aa.Vv. (2016d), "Utilización de las series de animación como recurso didáctico en la elaboración de casos. Análisis de una experiencia en la innovación docente en la Universitat Politècnica de València”, en INRED. II Congreso Nacional de Innovación Educativa y Docencia en Red, Universitat Politècnica de València, Valencia, pp. 9-20. Disponible en:

\section{http://ocs.editorial.upv.es/index.php/INRED/INRED2016/paper/viewFile/4430/2000}

(Consultado el 26 de mayo de 2019).

Aa.Vv. (2017), "La interactividad y dinamización en el aula mediante la implantación del diseño de casos prácticos jurídicos basados en series de animación”, 3C TIC, cuadernos de desarrollo aplicados a las TIC, núm. 4, pp. 1-18. Disponible en: http://www.3ciencias.com/wp-content/uploads/2016/12/ART1.pdf (Consultado el 02 de junio de 2019).

Aa.Vv. (2017a), "La utilización de un episodio de la serie de ficción «Los Simpson» como herramienta para la evaluación de la competencia transversal de pensamiento crítico en la Universitat Politècnica de València”, en Segundo Congreso Internacional Virtual sobre la Educación en el Siglo XXI, Universidad de Málaga, Málaga, pp. 560569. Disponible en: http://www.eumed.net/libros-gratis/actas/2017/educacion/index.htm (Consultado el 10 de junio de 2019). 
Aa.Vv. (2017b), "La evaluación en el ámbito jurídico de la competencia transversal pensamiento crítico mediante la utilización de series de ficción. Las TIC y las buenas prácticas en la docencia del Derecho, Huygens, Barcelona, pp. 191-201.

Aa.Vv. (2017c), "Evaluación de la competencia transversal CT09 Pensamiento crítico a través de series de ficción en la asignatura de Gestión empresarial de la edificación en el Máster en Edificación de la Universitat Politècnica de València”, en In-Red 2017-III Congreso Nacional de Innovación Educativa y Docencia en Red de la Universitat Politècnica de València, Universitat Politècnica de València, Valencia, pp. 1111-1120. Disponible en: http://ocs.editorial.upv.es/index.php/INRED/INRED2017/paper/viewFile/6901/3114 (Consultado el 10 de junio de 2019).

Álvarez Vigaray, R. (2006a), "El Derecho civil en el cuento "La Sirenita” de Andersen”, en Homenaje al profesor Lluis Puig i Ferriol, 1, Tirant lo Blanch, Valencia, pp. 75-80.

Álvarez Vigaray, R. (2006), "El Derecho civil en los cuentos de Andersen”, en Libro Homenaje al profesor Manuel Amorós Guardiola, 2, Colegio de Registradores de la Propiedad y Mercantiles de España, Madrid, pp. 3043-3053.

Cabedo Mallol, V., Casar Furió, M. E., Giménez Chornet, V., Oltra Gutiérrez, J. V. y Ramón Fernández, F. (2016), Casos prácticos jurídicos basados en series de animación, Tirant lo Blanch, Valencia.

Cabedo Mallol, V., Casar Furió, M. E., Giménez Chornet, V., Oltra Gutiérrez, J. V. y Ramón Fernández, F. (2017), Casos prácticos jurídicos basados en series de ficción, Tirant lo Blanch, Valencia.

Cabedo Mallol, V., Casar Furió, M. E., Giménez Chornet, V., Lull Noguera, C., Oltra Gutiérrez, J. V. y Ramón Fernández, F. (2018), Casos prácticos jurídicos basados en documentales, Tirant lo Blanch, Valencia.

Camilloni, A. (2019): "La enseñanza del derecho orientada al desarrollo de la creatividad", Revista pedagogía universitaria y didáctica del Derecho, vol. 6, núm. 1, pp. 5-22. Disponible en: https://pedagogiaderecho.uchile.cl/index.php/RPUD/article/view/53743/56409 (Consultado el 13 de julio de 2019). 
Canós Darós, L. y Ramón Fernández, F. (2005), “Aplicación de las nuevas tecnologías para el trabajo autónomo del alumno", en Actas del XX Simposium Nacional de la Unión Científica Internacional de Radio. URSI 2005, Universitat Politècnica de València, Gandía, pp. 1-4.

Canós Darós, L. y Ramón Fernández, F. (2007), "La aplicación de las TICs en el aprendizaje de disciplinas jurídicas aplicadas en carreras técnicas en el marco de la convergencia europea”, en Conocimiento Abierto, Sociedad Libre, Actas digitales del III Congreso ONLINE del Observatorio para la CiberSociedad, Observatorio para la Cibersociedad, Barcelona, pp. 1-7.

Canós Darós, L. y Ramón Fernández, F. (2009), "La calidad de la docencia respecto a las nuevas tecnologías y recursos didácticos”, Autogestión, cooperación y participación en las Ciencias Sociales, pp. 341-352.

Canós Daros, L., Ramón Fernández, F. y Albaladejo Fernández-Silgado, M. (2008), "Los roles docentes y discentes ante las nuevas tecnologías y el proceso de convergencia europea", en CD Resúmenes comunicaciones V Congreso Iberoamericano de Docencia Universitaria, AIDU e ICE, Valencia, pp. 388-390.

Capote Pérez, L. J. (2015), "Materiales audiovisuales de licencia libre para la docencia en Derecho civil", en Innovación en las enseñanzas universitarias: experiencias presentadas en las V Jornadas de Innovación educativa de la ULL, Universidad de La Laguna, La Laguna, pp. 62-77. Disponible en: https://www.ull.es/Private/folder/institucional/ull/calidad/innovacion/ebook_2015.pdf (Consultado el 10 de junio de 2019).

Cicero, N. K. (2018), “Innovar la enseñanza del derecho. ¿Solo se trata de Tecnologías de la Información y Comunicación?”, Revista pedagogía universitaria y didáctica del Derecho, vol. 5, núm. 2, pp. 91-109. Disponible en: https://pedagogiaderecho.uchile.cl/index.php/RPUD/article/view/51976/54571

(Consultado el 13 de julio de 2019).

Colao, P. A. (2016), “Definición de un método docente para el aprendizaje de la parte general del Derecho tributario por no juristas", Revista de Educación y Derecho = Education and law review, núm. 13, pp. 1-20. Disponible en: http://revistes.ub.edu/index.php/RED/article/view/15505/18662 (Consultado el 18 de junio de 2019). 
Cordero Cutillas, I., Escribano Tortajada, P. y Martínez Vázquez de Castro, L. (2014), "Cine y Derecho de Familia: una nueva visión de la metodología práctica", Revista de Educación y Derecho = Education and law review, núm. 9, 2014, pp. 1-16. Disponible en: http://revistes.ub.edu/index.php/RED/article/view/10209/13053 (Consultado el 18 de junio de 2019).

López Catalán, J. (2002), “Didáctica y tecnología. Una combinación justa en la creación del guión multimedia", Comunicación y pedagogía. Nuevas tecnologías y recursos didácticos (Primeras noticias), núm. 178, pp. 43-52.

Lucas-Santos, S. De (2017), "El uso de las TIC para el desarrollo de competencias con metodologías activas en Estadística Descriptiva del grado de ADE”, REDU. Revista de Docencia Universitaria, núm. 15, pp. 245-256. Disponible en: https://polipapers.upv.es/index.php/REDU/article/view/7405/9196 (Consultado el 02 de junio de 2019).

Marín Vichis, R. (2017), "Errores frecuentes en el uso de la metodología de la investigación jurídica", Revista pedagogía universitaria y didáctica del Derecho, vol. 4, núm.

2 ,

pp.

87-98.

Disponible

en:

https://pedagogiaderecho.uchile.cl/index.php/RPUD/article/view/47972/50527

(Consultado el 13 de julio de 2019).

Mercader, C. y Gairín Sallán, J. (2017), “Cómo utiliza el profesorado universitario las tecnologías digitales en sus aulas?", REDU. Revista de Docencia Universitaria, núm.
15 ,
pp.
257-273.
Disponible
en:

https://polipapers.upv.es/index.php/REDU/article/view/7635/9191 (Consultado el 03 de junio de 2019).

Navarro Frías, I. y Melero Bosch, L. (2018), "Trabajos de investigación de contenido jurídico en titulaciones de perfil no jurídico dentro de las ciencias sociales", Revista de Educación y Derecho = Education and law review, núm. 17, 2018, pp. 1-31. Disponible en: http://revistes.ub.edu/index.php/RED/article/view/21875/23501 (Consultado el 18 de junio de 2019).

Pérez Juan, J. A. (2014), "La enseñanza semipresencial y los recursos audiovisuales en historia del derecho", en Uso de las TIC en la docencia del derecho: aproximaciones docentes y metodológicas, Huygens, Barcelona, pp. 57-66. 
Pérez Vallejo, A. Ma . (2013), "Enseñanza práctica del Derecho civil: replanteamiento metodológico y experiencias de innovación", Revista de Educación y Derecho = Education and law review, núm. 8, pp. 1-17. Disponible en: http://revistes.ub.edu/index.php/RED/article/view/8023/9950 (Consultado el 18 de junio de 2019).

Ramón Fernández, F. (2012), El patrimonio cultural. Régimen legislativo y su protección, Tirant lo Blanch, Valencia.

Ramón Fernández, F. (2014), "La utilización de los módulos de aprendizaje en las asignaturas jurídicas: propuestas de mejora", en I Jornadas de Investigación de la Facultad de ADE, Universitat Politècnica de València, Valencia, pp. 112-119.

Ramón Fernández, F. (2014), “La utilización de las TICs en el estudio de casos en la asignatura de legislación patrimonial.", en Uso de las TIC en la docencia del Derecho: aproximaciones docentes y metodológicas, Huygens, Barcelona, pp. 197-210.

Ramón Fernández, F. y Saz Gil, Ma . I. (2004a), "La educación para la participación en el Espacio Europeo: la implantación del sistema de créditos europeos (Resumen de comunicación)", Revista Iberoamericana de Autogestión y Acción comunal, núm. 44, pp. 168-169.

Ramón Fernández, F. y Saz Gil, Ma. I. (2004b), "Las nuevas tecnologías como elemento facilitador de los métodos activos en la transmisión de competencias", en Hacia una docencia de calidad: políticas y experiencias. Actas del I Congreso de la Red Estatal de Docencia Universitaria y III Jornada de Mejora Educativa de la Universitat Jaume I, Universitat Jaume I, Castellón, pp. 330-341.

Ramos, G., Chiva, I. y Gómez, Ma. B. (2017), "Las competencias básicas en la nueva generación de estudiantes universitarios: Una experiencia de Innovación", REDU. Revista de Docencia Universitaria, núm. 15, pp. 37-55. Disponible en: https://polipapers.upv.es/index.php/REDU/article/view/5909/7947 (Consultado el 09 de junio de 2019).

Ruiz Sanz, M. (2014), "Instruir en Derecho y cine: una apuesta entre elecciones y pasiones", Revista de Educación y Derecho = Education and law review, núm. 9, pp. 1-

22. Disponible en: http://revistes.ub.edu/index.php/RED/article/view/10196/13049 (Consultado el 18 de junio de 2019). 
FRANCISCA RAMÓN FERNÁNDEZ. La enseñanza derecho civil a través del elemento audiovisual en titulaciones no jurídicas: una innovación docente en la Universitat Politècnica de València

$\underline{\text { Ruiz Sanz, M. (2010), "La enseñanza del Derecho a través del cine: implicaciones }}$ epistemológicas y metodológicas", Revista de Educación y Derecho $=$ Education and law review, núm. 2, pp. 1-16. Disponible en: http://revistes.ub.edu/index.php/RED/article/view/2320/2459 (Consultado el 18 de junio de 2019).

Urías Martínez, J. (1999), "El estudio de derecho a través de la creación de materiales audiovisuales", Revista de Enseñanza Universitaria, núm. 1, pp. 441-450. Disponible en: http://institucional.us.es/revistas/universitaria/extra1999/art_38.pdf (Consultado el 29 de mayo de 2019).

Verdera Izquierdo, B. (2014), "El aprendizaje y desarrollo del pensamiento jurídico a través de la jurisprudencia", Revista de Educación y Derecho = Education and law review, núm. $10, \quad$ pp. 1-22. Disponible en: http://revistes.ub.edu/index.php/RED/article/view/10711/13486 (Consultado el 18 de junio de 2019). 